\title{
Immunohistochemical and Transcriptional Analysis of SARS-CoV-2 Entry Factors and Renin-Angiotensin-Aldosterone System Components in Lethal COVID-19
}

\author{
Jasmin Dionne Haslbauer ${ }^{a}$ Anna Stalder ${ }^{a}$ Carl Zinner $^{b}$ Stefano Bassetti ${ }^{c}$ \\ Kirsten Diana Mertz ${ }^{d}$ Philip Went ${ }^{\mathrm{e}}$ Matthias Matter ${ }^{\mathrm{a}}$ Alexandar Tzankov ${ }^{\mathrm{a}}$ \\ apathology, Institute of Medical Genetics and Pathology, University Hospital Basel, University of Basel, Basel, \\ Switzerland; 'Department of Biomedicine, University of Basel, Basel, Switzerland; 'Division of Internal Medicine, \\ University Hospital Basel, University of Basel, Basel, Switzerland; ${ }^{d}$ Institute of Pathology, Cantonal Hospital \\ Baselland, Liestal, Switzerland; eInstitute of Pathology, Cantonal Hospital Graubünden, Chur, Switzerland
}

\section{Keywords}

Coronavirus disease $2019 \cdot$ Severe acute respiratory syndrome coronavirus- 2 - Renin-angiotensinaldosterone system · Angiotensin converting enzyme- 2 . Transmembrane protease serine subtype-2

\footnotetext{
Abstract

Introduction: Since angiotensin converting enzyme-2 (ACE2) was discovered as an essential entry factor of SARSCoV-2 (severe acute respiratory syndrome coronavirus-2), there has been conflicting evidence regarding the role of renin-angiotensin-aldosterone system (RAAS) in COVID-19. This study elucidates pulmonary expression patterns SARSCoV-2 entry factors (ACE2 and transmembrane protease serine subtype 2, TMPRSS2) and RAAS components in lethal COVID-19. Methods: Lung tissue from COVID-19 autopsies ( $n=$ 27) and controls $(n=23)$ underwent immunohistochemical staining for RAAS components (angiotensin receptors 1 and 2, ACE2 and Mas-receptor) and bradykinin receptors 1 and 2 . Staining of individual cellular populations (alveolar pneumocytes [ALV], desquamated cells [DES] and endothelium [END]) was measured by a binary scale (positive/negative).
}

SARS-CoV-2 was detected using immunohistochemistry against nucleocapsid protein, in-situ hybridization and quantitative reverse transcriptase polymerase chain reaction. Gene expression profiling for ACE2, ACE and TMPRSS2 was performed. Results: Subtle differences were observed when comparing COVID-19 patients and controls not reaching statistical significance, such as a higher incidence of ACE2-positivity in END ( $52 \%$ vs. $39 \%$ ) but lower positivity in ALVs (63\% vs. $70 \%)$ and an overall downregulation of ACE2 gene expression (0.25 vs. 0.55). However, COVID-19 patients with RAAS inhibitor (RAASi) intake had significantly shorter hospitalization times ( 5 vs. 12 days), higher viral loads $(57,517$ vs. $15,980 / 10^{6}$ RNase P-gene copies) and decreased ACE/ ACE2-expression ratios (4.58 vs. 11.07) than patients without. TMPRSS2 expression was significantly (1.76-fold) higher in COVID-19 patients than controls. Conclusion: Our study delineates the heterogeneous expression patterns of RAAS components in the lungs, which vary amongst cellular populations, and implies that COVID-19 patients with RAASi-intake present with a more rapid disease progression, although this requires further investigation.

(c) 2021 The Author(s)

Published by S. Karger AG, Basel karger@karger.com www.karger.com/pat

Karger $\stackrel{\text { ' }}{5}$

BOPEN ACCESS
(C) 2021 The Author(s)

Published by S. Karger AG, Basel

This is an Open Access article licensed under the Creative Commons Attribution-NonCommercial-4.0 International License (CC BY-NC) (http://www.karger.com/Services/OpenAccessLicense), applicable to the online version of the article only. Usage and distribution for commercial purposes requires written permission. 


\section{Introduction}

Shortly after the emergence of the COVID-19 pandemic, angiotensin converting enzyme-2 (ACE2) and transmembrane protease serine subtype-2 (TMPRSS2) were identified as requisite cellular entry factors for severe acute respiratory syndrome coronavirus-2 (SARSCoV-2) [1]. This promptly led to speculation regarding the role of the renin-angiotensin-aldosterone system (RAAS) in the pathophysiology of COVID-19 and raised questions whether RAAS inhibitor (RAASi) therapy, such as angiotensin converting enzyme inhibitors (ACEi), angiotensin receptor blockers (ARB) and aldosterone antagonists, predisposed to SARS-CoV-2 infection [2]. RAASi therapy [3] and underlying cardiovascular disease [4] have been previously shown to lead to an increased ACE2 expression, thus implying an increased susceptibility of viral entry in patients with cardiovascular comorbidities. Indeed, previous studies have shown a higher propensity of severe COVID-19 in patients with hypertension and underlying cardiovascular disease [5-9], further underscoring the need to analyze the pathophysiological link between SARS-CoV-2 and RAAS.

ACE2, a monocarboxypeptidase homologous to ACE, promotes the conversion of angiotensin II (Ang-II) to Ang-(1-7), both ligands of plasma membrane receptors AGTR1 and AGTR2 (Ang-II receptors 1/2) and MasR (Mas-receptor) which act as central regulators of blood pressure homeostasis [10]. In the lung, AGTR1 activation by Ang-II leads to vasoconstriction, fibrotic remodeling, angiogenesis and increased vascular permeability, whereas AGTR2 elicits vasodilation and downregulates fibrosis [11]. MasR activation by Ang-(1-7), concordantly to AGTR2, results in nitric oxide-dependent vasodilation, anti-fibrotic and antiangiogenic effects [10-12]. ACE2 thus acts as an antagonist to ACE, negatively regulating vascular tone and remodeling [13], and has been shown to inhibit acute lung injury/diffuse alveolar damage (ALI/DAD) in the pulmonary microenvironment $[14,15]$.

SARS-CoV-2 utilizes its spike protein to bind to ACE2. The spike protein is cleaved by a transmembrane serine protease, TMPRSS2, which facilitates membrane fusion [16], ultimately leading to ACE2 internalization and shedding $[13,14]$. Decreased membranous ACE2 leads to a relative accumulation of Ang-II, which then binds AGTR1, inducing vascular permeability and neutrophil accumulation; this induces noncardiogenic pulmonary edema and DAD $[14,15]$, a readily detected finding in
COVID-19 [9, 17]. Furthermore, decreased ACE2 prevents the degradation of des-Arg bradykinin, which results in the activation of bradykinin receptor 1 (BDKRB1), leading to hypotension, natriuresis and vasodilation due to the release of proinflammatory chemokines [18]. This "bradykinin storm" may be one of the underlying mechanisms for the inflammatory response in severe COVID-19 [19]. Thus, decreased membranous ACE2 may reduce susceptibility for viral entry but facilitates tissue damage, whereas increased ACE2 increases the likelihood for viral entry but simultaneously prevents ALI. These concepts demonstrate the complex, dynamic role of ACE2 in COVID-19 pathophysiology. To study the putative effects of RAAS and viral entry factors in severe COVID-19, we performed an immunohistochemical (IHC), viral in-situ analysis and gene expression profiling on RAAS components, bradykinin receptors, ACE2 and TMPRSS2 on lung tissue from patients with lethal COVID-19 versus age-matched controls.

\section{Materials and Methods}

\section{Patient Selection}

Twenty-seven COVID-19 autopsy cases and 23 controls were enrolled [9]. All COVID-19 patients tested positive for SARSCoV-2 per antemortem nasopharyngeal swab. The cause of death in all cases was COVID-19-associated respiratory failure. Agematched SARS-CoV-2 negative autopsy controls were selected in line with a range of etiologies (histomorphologically unremarkable lungs, $n=5$; pneumococcus pneumonia, $n=4$; influenza pneumonia, $n=5$; non-COVID-19 DAD, $n=9)$. Causes of nonCOVID-19 DAD included infections by other pathogens ( $E$. faecalis, C. albicans, K. pneumoniae and Pseudomonas spp.), postoperative/paraneoplastic and post-stem cell transplant/drug-induced. Controls with pneumococcus and influenza pneumonia did not have histological signs of DAD. ACEi, ARB, renin inhibitors and aldosterone antagonists were considered as RAASi.

Tissue Sampling, Processing, Immunohistochemistry, and in situ Hybridization

A lung tissue sample measuring $1.5 \times 1 \times 0.3 \mathrm{~cm}$ was placed in $4 \%$ phosphate-buffered formalin at autopsy for $48 \mathrm{~h}$ and embedded in paraffin. Tissue microarrays (TMAs) were generated of all samples using the TMA Grand Master (3DHistech Ltd., Budapest, Hungary). Representative regions of histological sequelae of COVID-19 (i.e., characteristic changes in the vascular compartment, DAD) were annotated by expert staff pathologists on corresponding hematoxylin and eosin-stained sections. These regions were transferred to a recipient block, generating three $1.5 \mathrm{~mm}$-diameter cores per patient, analogous to previous protocols [20]. Slides were stained with hematoxylin and eosin and for AGTR1\&2, ACE2, MasR, BDKRB1\&2 and SARS-CoV-2 nucleocapsid protein (online suppl. Table 1 for detailed protocols; for all online suppl. material, see www.karger.com/doi/10.1159/000520221). In-situ hybridization for SARS-CoV-2 was executed as previously described 
[21]. TMAs were manually assessed for IHC/in-situ hybridization positivity in different cellular populations (positive staining in alveolar pneumocytes [ALVs], desquamated cells [DESs] and endothelium [END]) by means of a binary scale (positive/negative) (online suppl. Methods). Results were independently controlled and corroborated by 2 pathologists (A.T. and J.D.H.) and Cohen's kappa coefficient $(\kappa)$ values ranging from fair $(\kappa=0.4$ for endothelial staining of AGTR2) to substantial agreement (e.g., $\kappa=0.769$ for alveolar and endothelial staining of ACE2) to perfect agreement (e.g., $\kappa=1$ for ACE2 staining DESs).

Quantitative Reverse Transcriptase Polymerase Chain Reaction See online supplementary Methods.

\section{Gene Expression Profiling}

See online supplementary Methods.

\section{Statistical Analysis}

Statistics was performed with $\mathrm{IBM}^{\circledR}$ SPSS $^{\circledR}$, version 25 (Armonk, NY, USA). 2-sided Fisher's exact tests were performed on categorical data. Nonparametric variables were computed using a
Mann-Whitney U-test, while parametric variables were processed with Student's $t$ test. Correlation analyses were performed with Spearman $\rho$. $p$ values below 0.05 were considered significant. No adjustment for multiple testing was applied because the setting was considered hypothesis generating/exploratory.

\section{Results}

\section{Clinical Characteristics and Pathology Findings}

Clinical characteristics are summarized in Table 1. A higher proportion of COVID-19 patients had a history of arterial hypertension compared to controls (85 vs. $62 \% ; p=$ n.s.); in parallel, the incidence of RAASi-intake was higher amongst COVID-19 patients (14/27; 52\% vs. $9 / 23 ; 39 \% ; p=$ n.s.). When comparing characteristics in lethal COVID-19, patients with RAASi-intake had a significantly shorter interval between admission and death

Table 1. Clinical characteristics of COVID-19 patients, classified according to RAASi-intake, in comparison with age-matched controls

\begin{tabular}{|c|c|c|c|c|}
\hline & \multicolumn{3}{|c|}{ COVID-19 patients $(n=27)$} & \multirow{2}{*}{$\begin{array}{l}\text { Controls } \\
(n=23)\end{array}$} \\
\hline & RAASi $(n=14)$ & no RAASi $(n=13)$ & total & \\
\hline \multicolumn{5}{|l|}{ Clinical characteristics } \\
\hline Age, median (IQR) & $80(21)$ & $72(12)$ & $78(33)$ & $76(35)$ \\
\hline BMI, median (IQR) & $27(9)$ & $28(5)$ & $27(19)$ & $27(13)$ \\
\hline Sex, male, $n(\%)$ & $10(77)$ & $10(77)$ & $19(70.4)$ & $13(56.5)$ \\
\hline Hospitalization time, days, median (IQR) & $5(4)^{* *}$ & $12(7)^{* *}$ & $7(27)$ & $7(39)$ \\
\hline Hypertension, $n(\%)$ & $14(100)^{* *}$ & $9(69)^{* *}$ & $23(85)$ & $15(65)$ \\
\hline Cardiovascular disease, $n$ (\%) & $9(64)$ & $9(69)$ & $18(67)$ & $14(61)$ \\
\hline \multicolumn{5}{|l|}{ Pathological findings } \\
\hline DAD (\% total) & $9(64)$ & $8(72)$ & $17(63)$ & $11(48)$ \\
\hline Exudative & $7(50)$ & $2(15)$ & $9(33)$ & $6(26)$ \\
\hline Proliferative & $2(14)$ & $6(46)$ & $8(27)$ & $5(22)$ \\
\hline None & $5(36)$ & $5(38)$ & $10(37)$ & $12(52)$ \\
\hline SARS-CoV-2 qRT-PCR viral load, mean (SD) & $57,517(64,451)^{* *}$ & $15,980(53,527)^{* *}$ & $37,517(64,161)$ & $\mathrm{n} / \mathrm{a}$ \\
\hline Expression of $A C E$, median (IQR) & $1.99(2.88)$ & $2.69(1.96)$ & $2.48(2.20)$ & $2.07(3.14)$ \\
\hline Expression of $A C E 2$, median (IQR) & $0.33(0.36)$ & $0.24(0.20)$ & $0.25(0.28)$ & $0.55(0.71)$ \\
\hline$A C E / A C E 2$ ratio, median (IQR) & $4.59(5.03)^{*}$ & $11.08(20.72)^{*}$ & $6.47(11.86)$ & $5.16(8.11)$ \\
\hline Expression of TMPRSS2, median (IQR) & $1.76(2.00)$ & $2.30(0.84)$ & $2.00(1.00)^{*}$ & $1.30(1.06)^{*}$ \\
\hline
\end{tabular}

RAASi, renin-angiotensin-aldosterone system inhibitor; DAD, diffuse alveolar damage; SARS-CoV-2, severe acute respiratory syndrome coronavirus-2; qRT-PCR, quantitative reverse transcriptase polymerase chain reaction; ACE/ACE2; angiotensin converting enzyme/angiotensin converting enzyme-2; TMPRSS2, transmembrane protease serine subtype $2 .{ }^{*}$ bold $=p$ values significant at the 0.05 level. ${ }^{*}$ bold $=$ $p$ values significant at the 0.01 level.

Fig. 1. Histomorphological characteristics of RAAS components and SARS-CoV-2 entry factors in lethal COVID-19 cases versus controls. Graphs display \% positivity in varying cellular populations. ${ }^{*}$ indicates $p$ values $<0.05$. Exemplary annotations: green $=$ positive, red = negative. Arrows: DESs/macrophages; asterisks: END; circles: ALVs. Error bars denote $100 \mu \mathrm{m}$. ALV, alveolar pneumocyte; DES, desquamated cell; END, endothelium; RAAS, renin-angiotensin-aldosterone system; SARS-CoV-2, severe acute respiratory syndrome coronavirus-2; AGTR1, angiotensin receptor 1; AGTR2, angiotensin receptor 2; MasR, Mas-receptor; ACE2, angiotensin converting enzyme-2; TMPRSS2, transmembrane protease serine subtype 2 .

(For figure see next page.) 


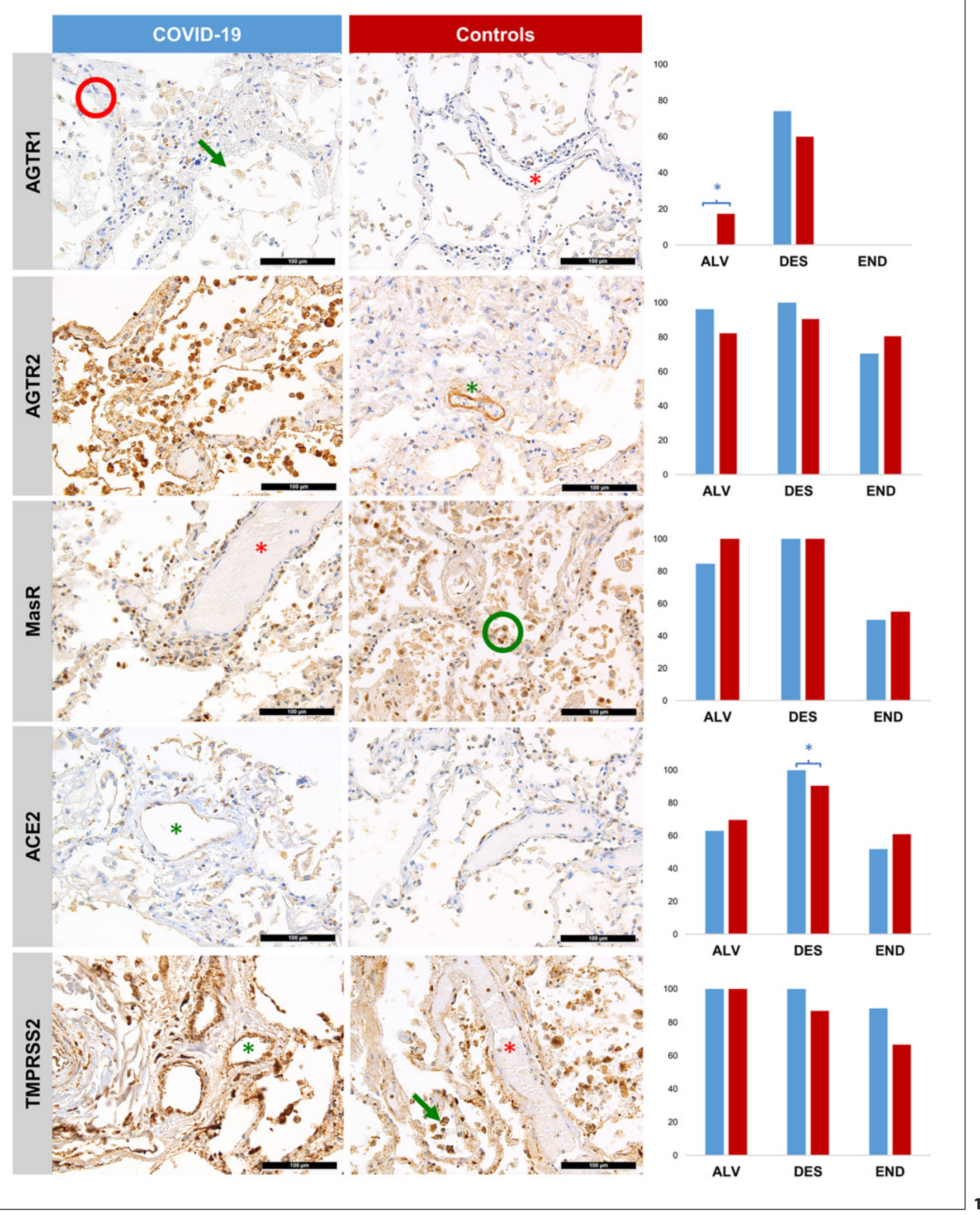

SARS-CoV-2 Entry Factors and RAAS in

Pathobiology 2022;89:166-177 DOI: $10.1159 / 00052022$ 
(hospitalization time 5 vs. 12 days; $p=0.006$ ) and, concordantly, displayed a significantly higher postmortem pulmonary viral load (57,517 vs. $15,980 / 10^{6} \mathrm{RPPH} 1 \mathrm{cop}-$ ies, $p=0.0041$; Fig. 3 ), mirroring results on the same autopsy cohort [22]. COVID-19 patients with RAASi-intake were more likely to present with exudative DAD (50\% vs. $15 \% ; p=$ n.s.). Furthermore, amongst COVID-19 patients, hospitalization time significantly negatively correlated with pulmonary viral load ( $\rho=-0.635$, $p=0.0004)$. As all COVID-19 cases stem from the first wave of the pandemic (March-June 2020), dexamethasone and antiviral treatments were uncommon (dexamethasone: $1 / 27$, remdesivir: $3 / 27$ ).

\section{Distribution of RAAS Components between COVID-19 Patients and Controls}

Figure 1 and Table 1 display histomorphological characteristics and trends of RAAS components and SARSCoV-2 entry factors between COVID-19 patients and controls. IHC for AGTR1 revealed weak membranous and cytoplasmatic positivity, predominantly in DESs (approximately half of epithelial and half of histiocytic origin) (up to $74 \%$ in COVID-19 lungs vs. $60 \%$ in controls, $p=$ n.s.). ALVs and END did not display any AGTR1 positivity in COVID-19 patients, while sporadic positivity was observed amongst controls $(4 / 23, p=0.04)$. In contrast, AGTR2 displayed more widespread staining, including ALVs, DESs and END (no differences between COVID-19 patients and controls). Staining for MasR revealed lower positivity in ALVs of COVID-19 patients than controls ( $82 \%$ vs. $100 \%, p=$ n.s.), while positivity in END was equally heterogeneous in both COVID-19 and control patients (48\% in both). Staining for ACE2 revealed similar positivity in ALVs in both COVID-19 patients and controls $(63 \%$ vs. $70 \%, p=$ n.s.), while a significantly higher number of cases displayed positivity in DESs (100 vs. $83 \%$; $p=0.04)$. Endothelial positivity of ACE2 was slightly but not significantly lower in COVID-19 patients (39 vs. $52 \%$, $p=$ n.s.), which was similarly mirrored in TMPRSS2 staining ( 85 vs. $61 \%, p=$ n.s.). In other cellular populations,

Fig. 2. Histomorphological characteristics of BDKRB1 and 2 in lethal COVID-19 cases versus controls. Annotations: green circle: membranous positivity of ALVs for BDKRB1; green arrow: membrano-cytoplasmatic positivity for BDKRB1 in a DES; red asterisk: positivity for BDKRB1 and 2 in interstitial cells including lymphocytes; green triangle: positivity for BDKRB2 in bronchial epithelium. Error bars denote $100 \mu \mathrm{m}$ for BDKRB1 and $200 \mu \mathrm{m}$ for BDKRB2. DES, desquamated cell; ALV, alveolar pneumocyte; BDKRB1 and 2, bradykinin receptors 1 and 2 .

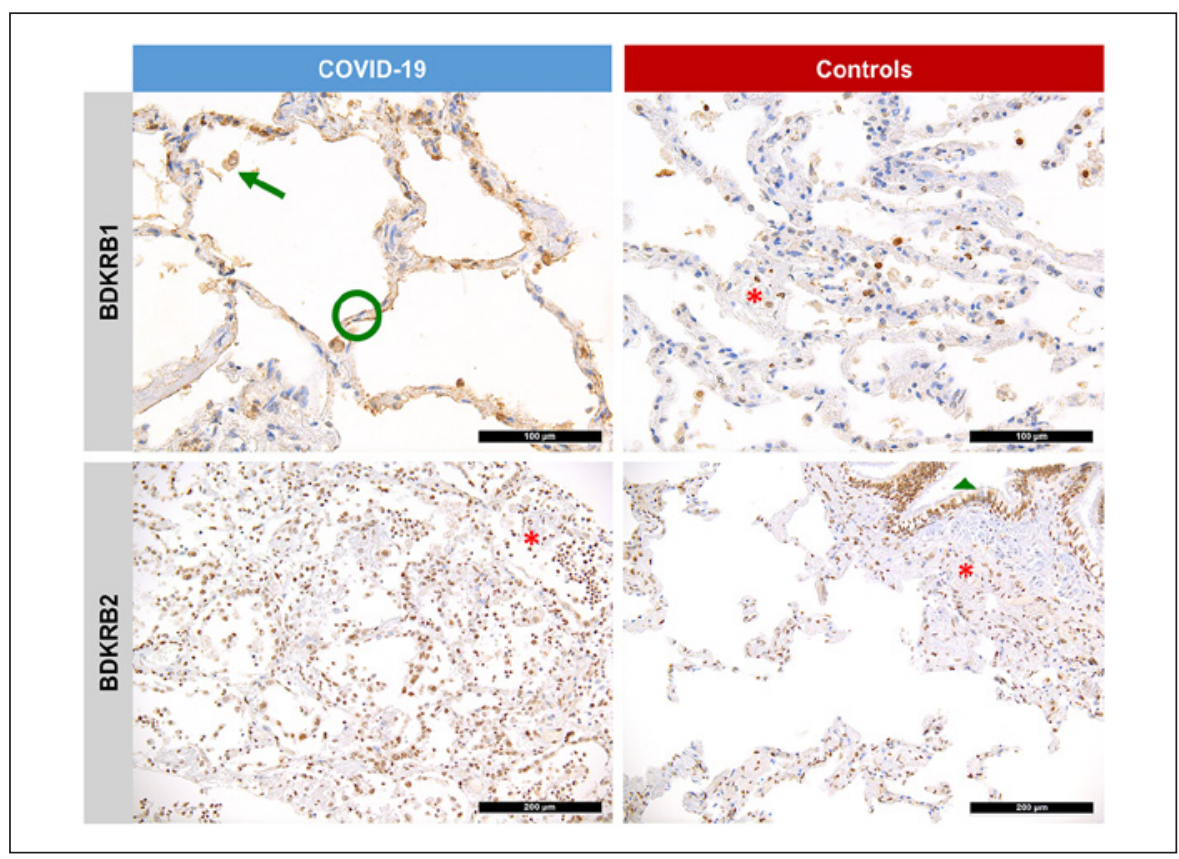

Fig. 3. Trends in \% positivity of RAAS components and SARSCoV-2 entry factors in lethal COVID-19 cases, grouped by intake of RAASi. A lower incidence of endothelial positivity of AGTR2, ACE2, and MasR is noted in cases with RAASi-intake. Conversely, ALV positivity is higher for ACE2 and MasR amongst cases with RAASi-intake. *indicates $p$ values $<0.05$. Viral load is mapped as per $10^{6}$ RPPH1 copies. ALV, alveolar pneumocyte; DES, desqua- mated cell; END, endothelium; RAASi, renin-angiotensin-aldosterone system inhibitor; SARS-CoV-2, severe acute respiratory syndrome coronavirus-2; AGTR1, angiotensin receptor 1; AGTR2, angiotensin receptor 2; ACE2, angiotensin converting enzyme-2; MasR, Mas-receptor; TMPRSS2, transmembrane protease serine subtype 2; RPPH1, RNase P-gene.

(For figure see next page.) 


\section{AGTR1}

100

80

60

40

20

64
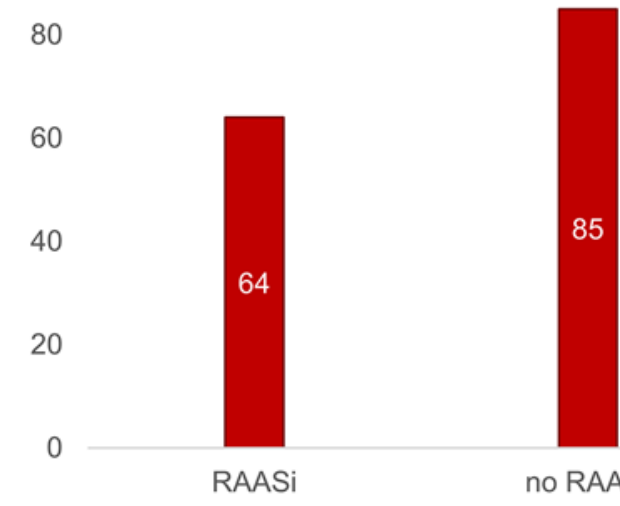

85

ACE2

100

80

60

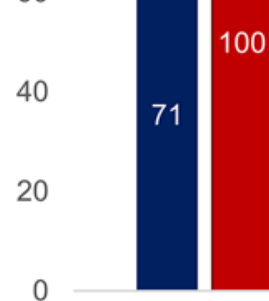

RAASi

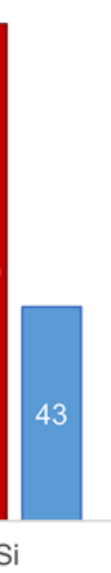

100
TMPRSS2
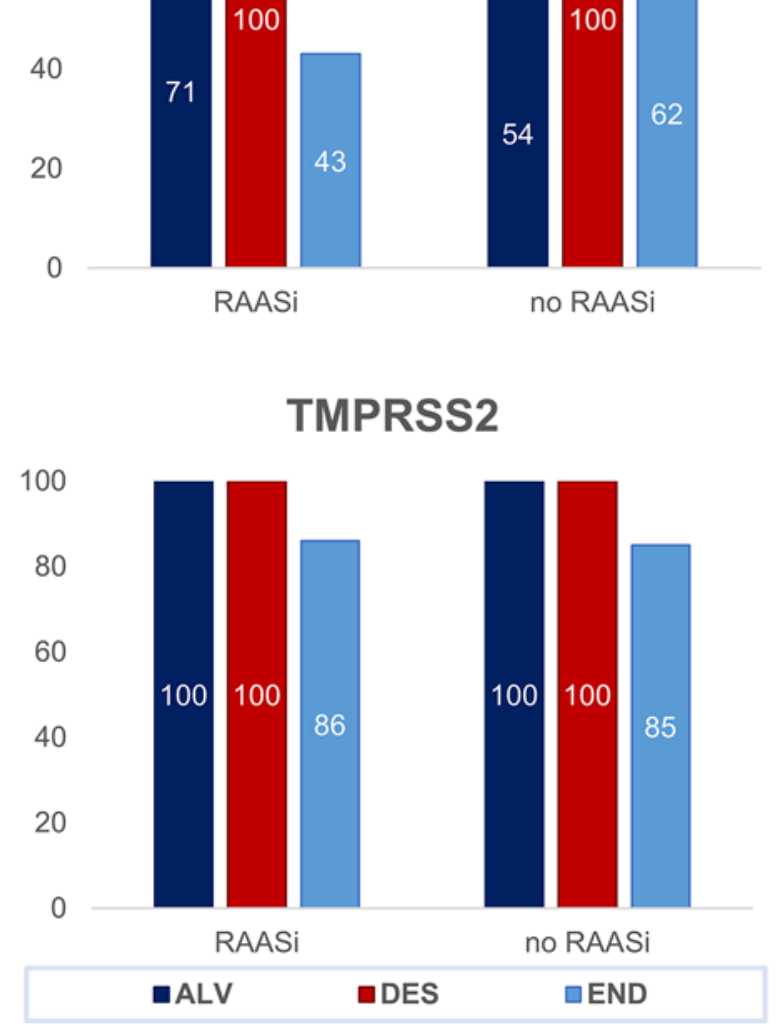

no RAASi
AGTR2

100

80

60

40

93
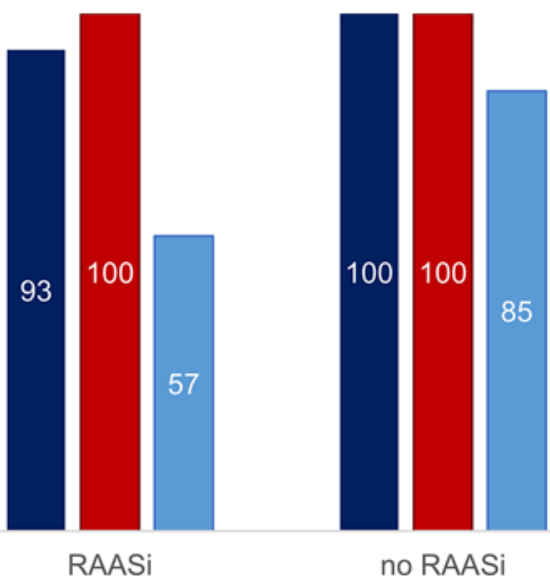

no RAASi

MasR

100

80

60

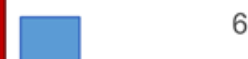

0

RAASi

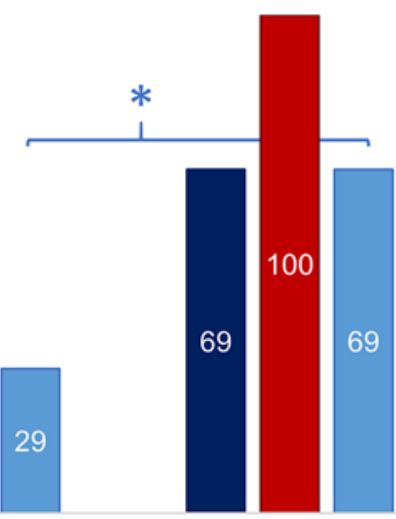

no RAASi

SARS-CoV-2 Viral Load

$10 \times 10^{4}$

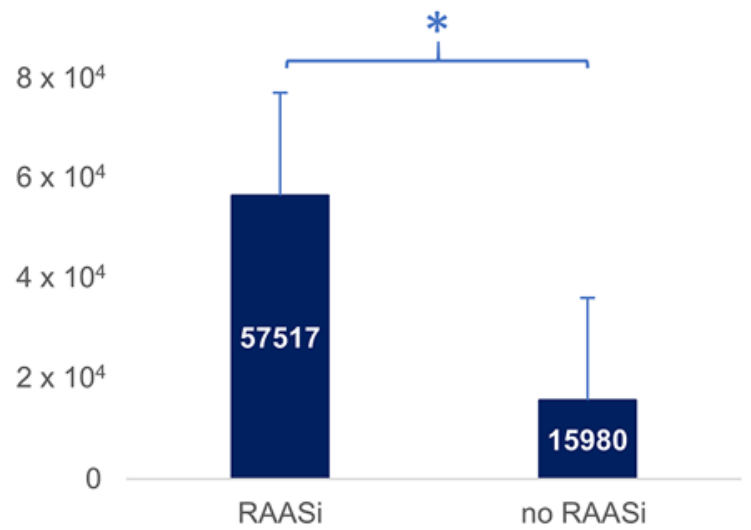


Fig. 4. Detection of SARS-CoV-2 N-protein by immunohistochemistry and RNA by ISH. Top left: positivity for viral N-protein in hyaline membranes and in the endothelial compartment; top right: positivity for viral RNA in ALVs, endothelia and hyaline membranes. Scale bars denote 100 $\mu \mathrm{m}$. Bottom: double staining with N-protein (red) and ERG (brown) reveals close association of viral proteins and the END (black arrows: endothelial cell surface coated with N-protein). SARS-CoV-2, severe acute respiratory syndrome coronavirus-2; $\mathrm{N}$-protein, nucleocapsid protein; ISH, insitu hybridization; END, endothelium; ALV, alveolar pneumocyte; ERG, ETS-related gene.

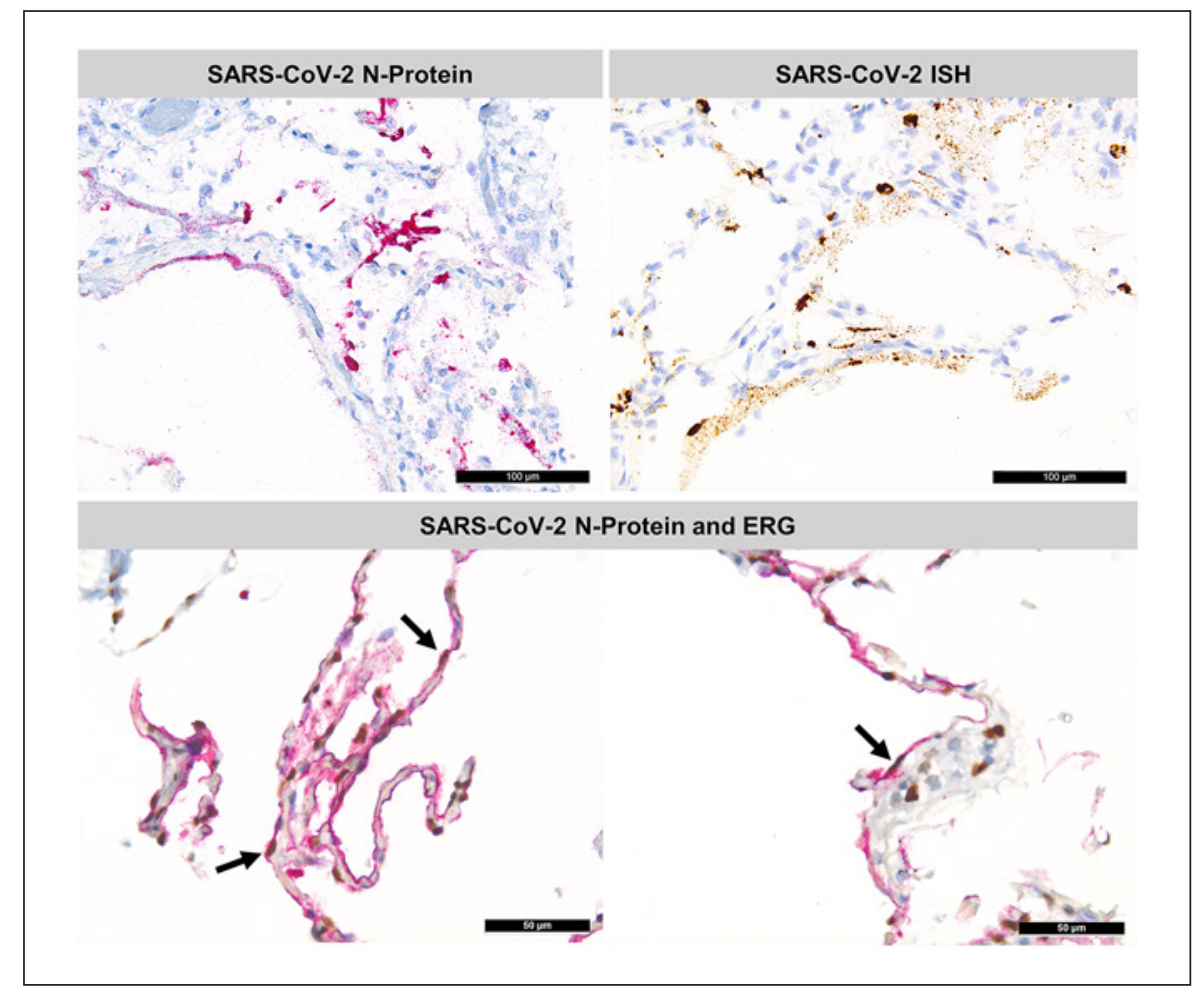

TMPRSS2 displayed equally widespread staining in both COVID-19 cases and controls. IHC for BDKRB1 and BDKRB2 revealed widespread nuclear and membranous positivity in ALVs and some DESs, as well as bronchial epithelium and immune cells such as granulocytes and lymphocytes, which in some COVID-19 cases revealed more extensive positivity, although this could not be quantified conclusively (Fig. 2).

Comparative Analysis of RAAS Components amongst COVID-19 Patients, Grouped by Intake of RAASi

Comparison of IHC positivity of individual cellular populations revealed characteristic trends amongst lethal COVID-19 cases dependent on the previous intake of RAASi (Fig. 3). This was predominantly discernible in the END, where there was a lower incidence of positivity amongst patients with RAASi-intake for AGTR2 (57 vs. $85 \%, p=$ n.s.), ACE2 (43 vs. $62 \%, p=$ n.s.), and, significantly, MasR ( 29 vs. $69 \%, p=0.04$ ). Positivity of ALVs behaved inversely, with a more prevalent incidence of positive staining for ACE2 and MasR in cases with RAASi-intake (ACE2: 71 vs. $62 \%, p=$ n.s.; MasR: 93 vs. $69 \%$, $p=$ n.s.). SARS-CoV-2 viral load was significantly higher in patients with RAASi-intake $\left(57,517\right.$ vs. $15,980 / 10^{6}$ RPPH1 copies, $p=0.0041$ ).
Detection of SARS-CoV-2 in COVID-19 Lung Tissue

Figure 4 displays the in-situ detection of SARS-CoV-2. SARS-CoV-2 was detected in various cellular populations $(1 / 27,4 \%$ in ALVs; $9 / 27,33 \%$ in DESs; and $3 / 27,11 \%$ in the END). Double staining with ERG (ETS-related gene) revealed viral nucleocapsid protein in close association with endothelial structures (ERG = brown and SARSCoV-2 N = red) (Fig. 4). SARS-CoV-2 viral load measured by quantitative reverse transcriptase polymerase chain reaction was significantly higher in cases staining positive ( 115,910 vs. $19,701 / 10^{6} \mathrm{RPPH} 1$ copies; $p=0.001$ ). Most notably, all cases with positive detection of SARSCoV-2 had a history of arterial hypertension with RAASi therapy.

\section{ACE/ACE2 Ratio and TMPRSS2 Gene Expression in COVID-19 Patients versus Controls Grouped by RAASi-Intake}

Gene expression of ACE, ACE2 and TMPRSS2 according to RAASi-intake is summarized in Figure 5. ACE/ACE2 ratio was slightly higher amongst COVID-19 patients than controls (6.47 vs. $5.16 ; p=$ n.s.). However, when grouped according to RAASi-intake, COVID-19 patients displayed significantly lower ACE/ACE2 ratios than those without RAASi ( 4.58 vs. $11.07 ; p=0.04$ ), while 
Fig. 5. Gene expression analysis of ACE/ ACE2 ratio and TMPRSS2 in COVID-19 versus controls. Left: patients with RAASiintake have a significantly lower ACE/ ACE2 ratio amongst COVID-19 patients $\left({ }^{*} p<0.05\right)$, while there was no significant difference in the control arm. Right: TMPRSS2 gene expression did not significantly differ with RAASi-intake. An overall significantly lower TMPRSS2 expression was observed in COVID-19 versus controls, irrespective of RAASi-intake $\left({ }^{*} p<0.05\right)$. Amongst patients without RAASi-intake, TMPRSS2 expression was higher in COVID-19 $\left({ }^{\dagger} p<0.01\right)$. ACE/ACE2, angiotensin converting enzyme/angiotensin converting enzyme-2; TMPRSS2, transmembrane protease serine subtype 2; COVID-19, coronavirus disease 2019; RAASi, reninangiotensin-aldosterone system inhibitor.
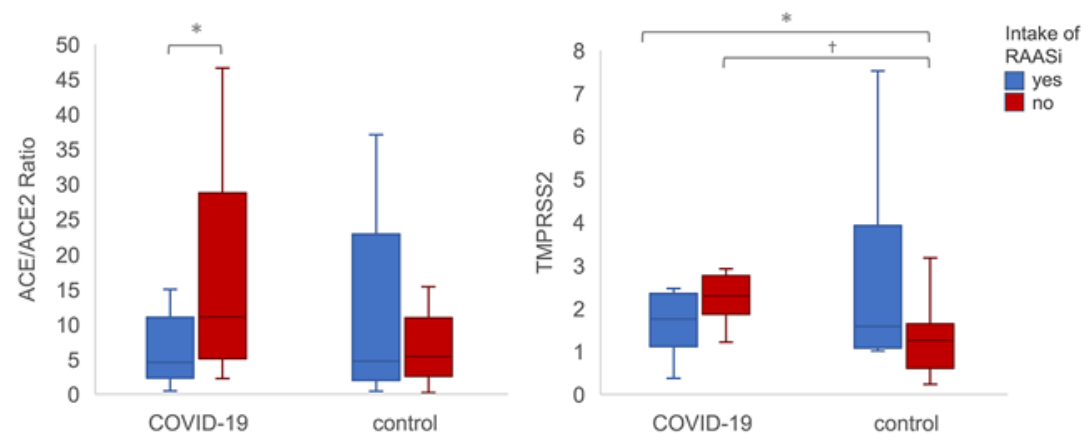

there was no significant difference in controls (4.73 vs. 5.38, $p=$ n.s.). ACE and ACE2 were decreased in COVID-19 patients versus controls, and even more decreased in cases with RAASi-intake, not achieving significance (Table 1). Overall TMPRSS2 expression was higher in COVID-19 patients than controls (1.76-fold; $p=0.04)$, in particular amongst patients without RAASiintake (1.83-fold; $p=0.003)$. Amongst COVID-19 cases, TMPRSS2- $(\rho=-0.629, p=0.001)$ and ACE-gene expression $(\rho=-0.629, p=0.001)$ strongly negatively correlated with viral load, while no significant correlation was observed for ACE2 (online suppl. Results); these correlations were not observed in controls.

\section{Discussion}

This analysis of SARS-CoV-2 entry factors and RAAS components revealed subtle differences between COVID-19 patients and controls, but more characteristic, significant RAASi-dependent differences amongst COVID-19 patients, both at the IHC and transcriptional level, which are systematically discussed below. ACE2 exhibited subtle nonsignificant lower positivity in ALVs and higher positivity in the END in COVID-19 patients versus controls. These findings, together with an overall downregulation of ACE2 gene expression, may indicate a redistribution of ACE2 in different cellular populations in COVID-19. ACE2 expression amongst sub-anatomical locations of the respiratory tract and cellular popula- tions has previously been reported as heterogeneous [2326 , with the most abundant expression in type 1 and, in particular, type 2 pneumocytes [26]. Our results support the premise of SARS-CoV-2-dependent ACE2 depletion, which may be the pathophysiological hallmark of COVID-19-associated DAD.

As described above, an overall paucity of ACE2 leads to accumulation of Ang-II and, by extension, an increased activity of AGTR1/AGTR2. Here, a slight, albeit nonsignificant increase of AGTR2 positivity amongst COVID-19 patients was observed, while AGTR1 staining showed weak staining; this is expected as AGTR2 has higher pulmonary tissue specificity $[27,28]$. In line with these observations, MasR, which is activated by ACE2 and Ang1-7, correspondingly displayed marginally lower positivity in ALVs and END of COVID-19 patients. The MasR/ACE2/Ang1-7-axis has a known protective role against ALI and chemokine production [29,30]; its downregulation thus contributes to a proinflammatory microenvironment, which may facilitate COVID-19-associated DAD [31].

TMPRSS2, which showed ubiquitous IHC positivity without significant morphological differences between COVID-19 patients and controls, strikingly presented with significantly higher gene expression in COVID-19. This may be explained by a higher enzymatic activity due to SARS-CoV-2-dependent internalization of ACE2 and thus supports our findings of decreased ACE2 in COVID-19. However, male gender-specific co-expression between TMPRSS2 and other genes re- 
lated to SARS-CoV-2 entry has been reported [32, 33], highlighting a possible explanation for the male predominance of COVID-19. This is further supported by the role of TMPRSS2-ERG fusions in prostate cancer [34] and the strong regulation of TMPRSS2 by androgens [35]. Thus, the male predominance amongst COVID-19 cases in our cohort (70.4\% vs. $56.5 \%)$ may similarly serve as an explanation for the increased $T M$ PRSS2 gene expression.

Comparison of RAAS component-staining as well as TMPRSS2 and ACE2 gene expression yielded characteristic findings dependent on RAASi-intake, summarized in Figure 6. Interestingly, COVID-19 patients with RAASi-intake had shorter hospitalization times, were more likely to present with exudative DAD and presented with higher pulmonary viral loads. Furthermore, all COVID-19 patients with positive viral detection suffered from arterial hypertension treated with RAASi. Therefore, RAASi may predispose to more severe disease dynamics, outcome and extensive viral replication, leading to earlier death. This may conceivably be due to RAASidependent increase of ACE2, which was previously described in the literature $[3,36,37]$, possibly also reflected by observations on ALVs in this study as well as the significantly decreased $A C E / A C E 2$ ratio in COVID-19 patients taking RAASi. Indeed, a decreased $A C E / A C E 2$ ratio has previously been observed in ARDS [38]. Additionally, the findings of this study mirror analyses performed on the same cohort, linking differing immunopathological [22] and cardiopathological [39] characteristics to hospitalization time and viral load; whether this is connected to changes in RAAS remains to be elucidated. In contrast to our observations however, a series of population-based studies have refuted any associations between RAASi and unfavorable prognosis of COVID-19 [40-43], although one study reported a higher risk of hospitalization among COVID-19 patients taking RAASi (OR 1.84) or ARB (OR 1.61) [40]. Another prospective randomized trial reported no significant difference of disease severity upon discontinuation of RAASi but suggests that discontinuation may lead to faster recovery [44]. Our data should thus be interpreted with caution, especially because of several potential unmeasured confounding factors and the limited statistical power of a smaller cohort.

Mounting evidence suggests that the endothelium plays an instrumental role in COVID-19 [31], characterized by an upregulation of thromboinflammatory genes $[17,45]$ leading to increased incidence of (micro-)thrombotic events [46]. Endothelial tropism for SARS-CoV-2 was observed in this study, further underlining these

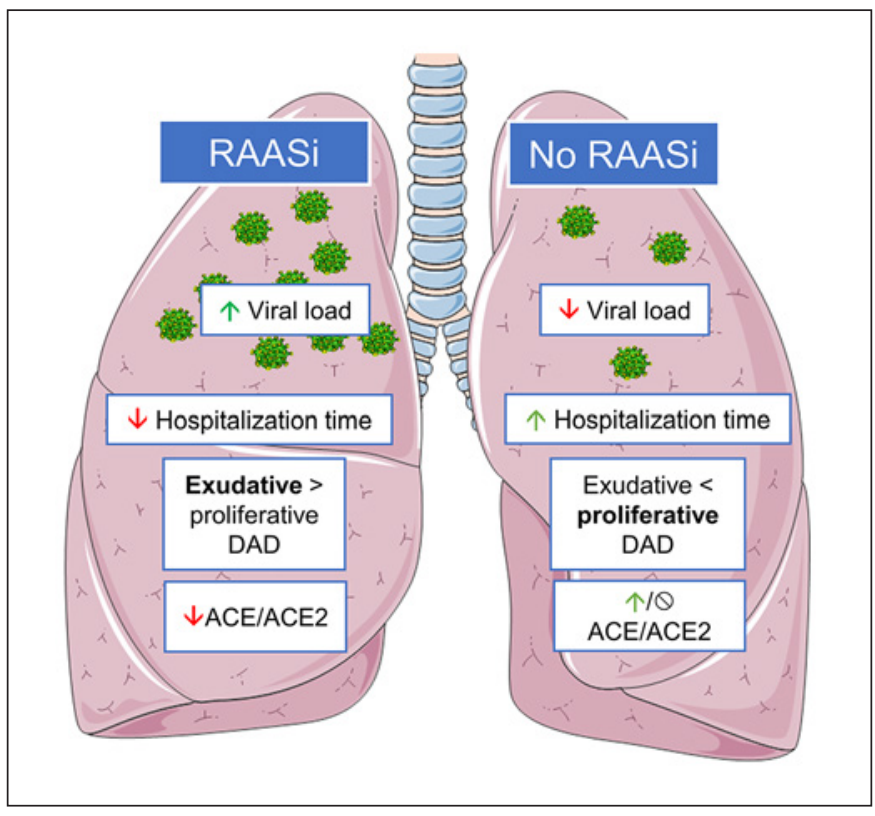

Fig. 6. Summary of clinical and pathological characteristics in lethal COVID-19, dependent on RAASi-intake. Patients with RAASi-intake were observed to have higher viral loads, shorter hospitalization times (time till death) and significantly lower ACE/ ACE2 ratios. A higher proportion of patients with RAASi-intake presented with exudative DAD, in line with the shorter hospitalization times. Original lung artwork by Servier Medical Art $@$, licensed under a Creative Commons Attribution 3.0 Unported License. COVID-19, coronavirus disease 2019; RAASi, renin-angiotensin-aldosterone system inhibitor; ACE/ACE2, angiotensin converting enzyme/angiotensin converting enzyme-2; DAD, diffuse alveolar damage.

findings. Interestingly, endothelial cells expressed less ACE2 and MasR under RAASi, while ALVs expressed more. These findings may be significant, taking into consideration the pathophysiological ties between RAAS and vascular inflammation and remodeling [47]. Previously performed in-vitro studies demonstrated an upregulated ACE2/MasR axis and increased ADAM17-mediated shedding of ACE2 in CD34+ hematopoietic stem/progenitor cells under hypoxic stimulation. These effects were mirrored by increase of vascular endothelial growth factor and stromal-derived factor $1 \alpha$, implying a crucial role of ACE2/MasR in vascular repair [48]. Thus, a decreased activity of ACE/MasR in COVID-19 cases of our cohort may signify a dysregulation in vascular signaling and remodeling in addition to thromboinflammation. Further studies of RAAS expression patterns within the endothelium in particular and the associated expression patterns in other cellular populations in the pulmonary microenvironment are required. 
The complex interaction between the Kallikrein-Kinin system and RAAS is well established. ACEi have previously been found to increase bradykinin synthesis, thus stimulating BDKRB1/2, which, in turn, results in an upregulation of endothelial nitric oxide synthase, angiogenesis-related genes and prostaglandins, while simultaneously downregulating NF-кB [19, 49]. Diffuse positivity of inflammatory cells (mostly granulocytes, lymphocytes, and macrophages) for ACE2 and BDKRB1/2 in our cohort potentially supports this. However, staining patterns for BDKRB1/2 were diffuse in all samples, which rendered difficulties in statistical analysis between COVID-19 patients and controls. Thus, although this study does not offer any histological evidence of a "bradykinin storm," its inherent role in severe COVID-19 needs to be further elucidated.

This study has several limitations. Firstly, its retrospective design may give rise to confounding factors which may have influenced the expression of $A C E$ and $A C E 2$. Its limited sample size $(n=50)$ suggests that results may not be representative enough and could explain why some $p$ values did not reach significance. Secondly, controls were pooled from a wide variety of histological subgroups, all of which likely possess extensive variations in RAAS component expression. Thirdly, several COVID-19 patients without RAASi therapy (69\%) suffered from arterial hypertension; these are patients who were treated with other antihypertensive drugs; the extent of which these impact RAAS is currently unclear. Fourthly, the heterogeneity of lung tissue is likely not sufficiently captured with the TMA. Lastly, IHC positivity was only measured by a binary scale as staining intensity of diaminobenzidene does not reflect antigen quantity. In summary, our findings underscore the heterogeneity of the expression of RAAS components described in the literature and identify a potential link between RAASi-intake and disease dynamics in COVID-19, which urgently requires further investigation.

\section{Acknowledgments}

We would like to thank all patients included in this study and their relatives, as well Susi Grieshaber, Michèle Baumann and Petra Hirschmann for their expertise in immunohistochemistry.

\section{Statement of Ethics}

This study has received approval by the Ethics Committee of Northwestern and Central Switzerland (ID 2020-00629). Samples used in this study were obtained as part of routine medical care. Written informed consent was obtained from participants prior to the study (or next of kin).

\section{Conflict of Interest Statement}

The authors have no financial interests to disclose. A.T. is a coeditor of Pathobiology but withdrew in all respective functions in connection to the present manuscript.

\section{Funding Sources}

This study was supported by the Botnar Research Centre for Child Health.

\section{Author Contributions}

Study design was contributed by A.T. and J.D.H. J.D.H. and A.T. completed the manuscript. Clinical data acquisition was contributed by J.D.H. and A.S. Histology was contributed by J.D.H., M.M., and A.T. Statistics was contributed by J.D.H. Critical revision of the manuscript was contributed by A.S., S.B., K.M., C.Z., and M.M. All authors read and approved of the manuscript.

\section{Data Availability Statement}

All data generated or analyzed during this study are included in this article and its online supplementary Material files. Further enquiries can be directed to the corresponding author.

\section{References}

1 Hoffmann M, Kleine-Weber H, Schroeder S, Krüger N, Herrler T, Erichsen S, et al. SARSCoV-2 cell entry depends on ACE2 and TMPRSS2 and is blocked by a clinically proven protease inhibitor. Cell. 2020 Apr;181(2): 271-80.e8.

2 Fang L, Karakiulakis G, Roth M. Are patients with hypertension and diabetes mellitus at increased risk for COVID-19 infection? Lancet Respir Med. 2020 Apr;8(4):e21.

3 Ferrario CM, Jessup J, Chappell MC, Averill DB, Brosnihan KB, Tallant EA, et al. Ef- fect of angiotensin-converting enzyme inhibition and angiotensin II receptor blockers on cardiac angiotensin-converting enzyme 2. Circulation. 2005 May;111(20): 2605-10.

4 Epelman S, Tang WH, Chen SY, Van Lente F, Francis GS, Sen S. Detection of soluble angiotensin-converting enzyme 2 in heart failure: insights into the endogenous counter-regulatory pathway of the renin-angiotensin-aldosterone system. J Am Coll Cardiol. 2008 Aug: 52(9):750-4.
5 Yang J, Zheng Y, Gou X, Pu K, Chen Z, Guo Q, et al. Prevalence of comorbidities and its effects in patients infected with SARS-CoV-2: a systematic review and meta-analysis. Int J Infect Dis. 2020 May;94: 91-5.

6 Chen N, Zhou M, Dong X, Qu J, Gong F, Han Y, et al. Epidemiological and clinical characteristics of 99 cases of 2019 novel coronavirus pneumonia in Wuhan, China: a descriptive study. Lancet. 2020 Feb; 395(10223):507-13. 
7 Wang D, Hu B, Hu C, Zhu F, Liu X, Zhang J, et al. Clinical characteristics of 138 hospitalized patients with 2019 novel coronavirus-infected pneumonia in Wuhan, China. JAMA. 2020 Mar;323(11):1061-9.

8 Inciardi RM, Lupi L, Zaccone G, Italia L, Raffo $\mathrm{M}$, Tomasoni $\mathrm{D}$, et al. Cardiac involvement in a patient with coronavirus disease 2019 (COVID-19). JAMA Cardiol. 2020 Jul;5(7): 819-24.

9 Menter T, Haslbauer JD, Nienhold R, Savic S, Hopfer H, Deigendesch N, et al. Postmortem examination of COVID-19 patients reveals diffuse alveolar damage with severe capillary congestion and variegated findings in lungs and other organs suggesting vascular dysfunction. Histopathology. 2020;77:198-209.

10 Deshotels MR, Xia H, Sriramula S, Lazartigues E, Filipeanu CM. Angiotensin II mediates angiotensin converting enzyme type 2 internalization and degradation through an angiotensin II type I receptor-dependent mechanism. Hypertension. 2014 Dec;64(6): 1368-75.

11 Catarata MJ, Ribeiro R, Oliveira MJ, Robalo Cordeiro C, Medeiros R. Renin-angiotensin system in lung tumor and microenvironment interactions. Cancers. 2020 Jun;12(6):1457.

12 Sampaio WO, Souza dos Santos RA, FariaSilva R, da Mata Machado LT, Schiffrin EL, Touyz RM. Angiotensin-(1-7) through receptor mas mediates endothelial nitric oxide synthase activation via Akt-dependent pathways. Hypertension. 2007 Jan;49(1): 185-92.

13 Kuba K, Imai Y, Rao S, Gao H, Guo F, Guan $\mathrm{B}$, et al. A crucial role of angiotensin converting enzyme 2 (ACE2) in SARS coronavirusinduced lung injury. Nat Med. 2005 Aug; 11(8):875-9.

14 Imai Y, Kuba K, Rao S, Huan Y, Guo F, Guan $\mathrm{B}$, et al. Angiotensin-converting enzyme 2 protects from severe acute lung failure. Nature. 2005;436(7047):112-6.

$15 \mathrm{Gu} \mathrm{H}$, Xie Z, Li T, Zhang S, Lai C, Zhu P, et al. Angiotensin-converting enzyme 2 inhibits lung injury induced by respiratory syncytial virus. Sci Rep. 2016 Jan;6:19840.

16 Heurich A, Hofmann-Winkler H, Gierer S, Liepold T, Jahn O, Pöhlmann S. TMPRSS2 and ADAM17 cleave ACE2 differentially and only proteolysis by TMPRSS2 augments entry driven by the severe acute respiratory syndrome coronavirus spike protein. J Virol. 2014 Jan;88(2):1293-307.

17 Ackermann M, Verleden SE, Kuehnel M, Haverich A, Welte T, Laenger F, et al. Pulmonary vascular endothelialitis, thrombosis, and angiogenesis in covid-19. N Engl J Med. 2020 Jul;383(2):120-8.

18 Sodhi CP, Wohlford-Lenane C, Yamaguchi Y, Prindle T, Fulton WB, Wang S, et al. Attenuation of pulmonary ACE2 activity impairs inactivation of des-Arg9 bradykinin/ BKB1R axis and facilitates LPS-induced neutrophil infiltration. Am J Physiol Lung Cell Mol Physiol. 2018 Jan;314(1):L17-31.
19 Garvin MR, Alvarez C, Miller JI, Prates ET, Walker AM, Amos BK, et al. A mechanistic model and therapeutic interventions for COVID-19 involving a RAS-mediated bradykinin storm. Elife. 2020 Jul;9:e59177.

20 Battifora $\mathrm{H}$. The multitumor (sausage) tissue block: novel method for immunohistochemical antibody testing. Lab Invest. 1986 Aug; 55(2):244-8.

21 Reinhold A, Tzankov A, Matter M, MihicProbst D, Scholl HPN, Meyer P. Ocular pathology and occasionally detectable intraocular SARS-CoV-2 RNA in five fatal COVID-19 cases. Ophthalmic Res. 2021;64(5):785-92.

22 Nienhold R, Ciani Y, Koelzer VH, Tzankov A, Haslbauer JD, Menter T, et al. Two distinct immunopathological profiles in autopsy lungs of COVID-19. Nat Commun. 2020 Oct; 11(1):5086.

23 Butowt R, Bilinska K. SARS-CoV-2: olfaction, brain infection, and the urgent need for clinical samples allowing earlier virus detection. ACS Chem Neurosci. 2020 May;11(9):12003.

24 Sungnak W, Huang N, Bécavin C, Berg M, Queen R, Litvinukova M, et al. SARS-CoV-2 entry factors are highly expressed in nasal epithelial cells together with innate immune genes. Nat Med. 2020 May;26(5):681-7.

25 Hamming I, Timens W, Bulthuis ML, Lely AT, Navis G, van Goor H. Tissue distribution of ACE2 protein, the functional receptor for SARS coronavirus. A first step in understanding SARS pathogenesis. J Pathol. 2004 Jun; 203(2):631-7.

26 Zhao Y, Zhao Z, Wang Y, Zhou Y, Ma Y, Zuo W. Single-cell RNA expression profiling of ACE2, the receptor of SARS-CoV-2. Am J Respir Crit Care Med. 2020 Sep;202(5):756-9.

27 Bullock GR, Steyaert I, Bilbe G, Carey RM, Kips J, De Paepe B, et al. Distribution of type1 and type- 2 angiotensin receptors in the normal human lung and in lungs from patients with chronic obstructive pulmonary disease. Histochem Cell Biol. 2001 Feb;115(2):11724.

28 Königshoff M, Wilhelm A, Jahn A, Sedding $\mathrm{D}$, Amarie OV, Eul B, et al. The angiotensin II receptor 2 is expressed and mediates angiotensin II signaling in lung fibrosis. Am J Respir Cell Mol Biol. 2007 Dec;37(6):64050.

29 Li Y, Zeng Z, Li Y, Huang W, Zhou M, Zhang $\mathrm{X}$, et al. Angiotensin-converting enzyme inhibition attenuates lipopolysaccharide-induced lung injury by regulating the balance between angiotensin-converting enzyme and angiotensin-converting enzyme 2 and inhibiting mitogen-activated protein kinase activation. Shock. 2015 Apr;43(4):395-404.

30 Li Y, Cao Y, Zeng Z, Liang M, Xue Y, Xi C, et al. Angiotensin-converting enzyme 2 /angiotensin-(1-7)/Mas axis prevents lipopolysaccharide-induced apoptosis of pulmonary microvascular endothelial cells by inhibiting JNK/NF-кB pathways. Sci Rep. 2015 Feb;5: 8209 .
31 Bösmüller H, Matter M, Fend F, Tzankov A. The pulmonary pathology of COVID-19. Virchows Arch. 2021 Jan;478(1):137-50.

32 Piva F, Sabanovic B, Cecati M, Giulietti M. Expression and co-expression analyses of TMPRSS2, a key element in COVID-19. Eur J Clin Microbiol Infect Dis. 2021 Feb;40(2): 451-5.

33 Baratchian M, McManus JM, Berk MP, Nakamura F, Mukhopadhyay S, Xu W, et al. Androgen regulation of pulmonary AR, TMPRSS2 and ACE2 with implications for sexdiscordant COVID-19 outcomes. Sci Rep. 2021 May;11(1):11130.

34 Tomlins SA, Laxman B, Varambally S, Cao X, $\mathrm{Yu} J$, Helgeson $\mathrm{BE}$, et al. Role of the TMPRSS2-ERG gene fusion in prostate cancer. Neoplasia. 2008 Feb;10(2):177-88.

35 Clinckemalie L, Spans L, Dubois V, Laurent $\mathrm{M}$, Helsen C, Joniau S, et al. Androgen regulation of the TMPRSS2 gene and the effect of a SNP in an androgen response element. Mol Endocrinol. 2013 Dec;27(12):2028-40.

36 Abuohashish HM, Ahmed MM, Sabry D, Khattab MM, Al-Rejaie SS. ACE-2/Ang1-7/ Mas cascade mediates ACE inhibitor, captopril, protective effects in estrogen-deficient osteoporotic rats. Biomed Pharmacother. 2017 Aug;92:58-68.

37 Ye R, Liu Z. ACE2 exhibits protective effects against LPS-induced acute lung injury in mice by inhibiting the LPS-TLR4 pathway. Exp Mol Pathol. 2020 Apr;113:104350.

38 Wösten-van Asperen RM, Lutter R, Specht PA, Moll GN, van Woensel JB, van der Loos $\mathrm{CM}$, et al. Acute respiratory distress syndrome leads to reduced ratio of ACE/ ACE2 activities and is prevented by angiotensin-(1-7) or an angiotensin II receptor antagonist. J Pathol. 2011 Dec;225(4):61827.

39 Haslbauer JD, Tzankov A, Mertz KD, Schwab N, Nienhold R, Twerenbold R, et al. Characterisation of cardiac pathology in 23 autopsies of lethal COVID-19. J Pathol Clin Res. 2021; 7(4):326-37.

40 Mehta P, McAuley DF, Brown M, Sanchez E, Tattersall RS, Manson JJ. COVID-19: consider cytokine storm syndromes and immunosuppression. Lancet. 2020;395(10229):10334.

41 Reynolds HR, Adhikari S, Pulgarin C, Troxel $\mathrm{AB}$, Iturrate $\mathrm{E}$, Johnson $\mathrm{SB}$, et al. Renin-angiotensin-aldosterone system inhibitors and risk of COVID-19. N Engl J Med. 2020; 382(25):2441-8.

42 de Abajo FJ, Rodríguez-Martín S, Lerma V, Mejía-Abril G, Aguilar M, García-Luque A, et al. Use of renin-angiotensin-aldosterone system inhibitors and risk of COVID-19 requiring admission to hospital: a case-population study. The Lancet. 2020 May;395(10238): 1705-14.

43 Mancia G, Rea F, Ludergnani M, Apolone G, Corrao G. Renin-angiotensin-aldosterone system blockers and the risk of COVID-19. N Engl J Med. 2020;382(25):2431-40. 
44 Bauer A, Schreinlechner M, Sappler N, Dolejsi $\mathrm{T}$, Tilg $\mathrm{H}$, Aulinger BA, et al. Discontinuation versus continuation of renin-angiotensin-system inhibitors in COVID-19 (ACEICOVID): a prospective, parallel group, randomised, controlled, open-label trial. Lancet Respir Med. 2021;9(8):863-72.

45 Gu SX, Tyagi T, Jain K, Gu VW, Lee SH, Hwa JM, et al. Thrombocytopathy and endotheliopathy: crucial contributors to COVID-19 thromboinflammation. Nat Rev Cardiol. 2021 Mar;18(3):194-209.
46 Bilaloglu S, Aphinyanaphongs Y, Jones S, Iturrate E, Hochman J, Berger JS. Thrombosis in hospitalized patients with COVID-19 in a New York city health system. JAMA. 2020 Aug;324(8):799-801.

47 Pacurari M, Kafoury R, Tchounwou PB, Ndebele $K$. The renin-angiotensin-aldosterone system in vascular inflammation and remodeling. Int J Inflam. 2014;2014:689360.
48 Joshi S, Wollenzien H, Leclerc E, Jarajapu YP. Hypoxic regulation of angiotensin-converting enzyme 2 and mas receptor in human CD34+ cells. J Cell Physiol. 2019 Nov;234(11): 20420-31.

49 Li P, Kondo T, Numaguchi Y, Kobayashi K, Aoki M, Inoue N, et al. Role of bradykinin, nitric oxide, and angiotensin II type 2 receptor in imidapril-induced angiogenesis. Hypertension. 2008 Feb;51(2):252-8. 